\title{
LECTURAS EDUCATIVAS DE LA TELEVISIÓN. ESTUDIO DE LA MÚSICA DE UNA MUESTRA DE CABECERAS Y BÚSQUEDA DE PROPUESTAS EDUCATIVAS ${ }^{1}$
}

\author{
Amparo Porta ${ }^{2}$
}

\begin{abstract}
This work is part of a larger international research project conducted to examine television programmes from Brazil, Argentina, Chile and Spain. The article presents the study carried out to analyse the opening themes used in Spanish children's programmes. The aim was to determine the main motivation for and musical contents of television productions, their preferences, common elements and tendencies. To achieve this objective, the study examines the most prominent song in the programme, that is to say, the one used to present the programme. The findings show that the music employed in these opening themes undergoes a steady and substantial process of loss of cultural identity, and of the values of diversity and difference. Finally, the article outlines, as a suggestion that could be useful in the search for educational alternatives, an interdisciplinary educational project: the creation of a "City of sound".
\end{abstract}

Keywords: analysis; children; music education; listening; observation; soundtrack; television

Resúmen: El trabajo que presentamos forma parte de un proyecto de investigación internacional realizado con programaciones televisivas de Brasil, Argentina, Chile y España. En el texto se presenta el estudio realizado sobre las cabeceras de programaciones infantiles españolas. La finalidad ha sido conocer los ejes generadores y contenidos musicales de las producciones televisivas, sus preferencias, elementos comunes y tendencias. Todo ello por medio de su canción más destacada, la de presentación del programa. Los resultados obtenidos indican que la música de estas cabeceras sufre un alto y constante proceso de pérdida de la identidad cultural, los valores de la diversidad y de la diferencia. El texto muestra, finalmente, como apunte para la búsqueda de alternativas educativas, un proyecto educativo interdisciplinar: la creación de una ciudad sonora.

Palabras clave: niños, educación musical, escucha, observación, banda sonora, televisión

\section{Planteamiento del problema}

La educación tiene como uno de sus fines preparar a los sujetos para enfrentar los desafíos de su época (Giroux, 1991). Por ello el paisaje sonoro de la televisión requiere ser considerado en el debate educativo, pero, para que ello sea posible, existe una clara

Porta, A. (2012). Lecturas educativas de la televisión. Estudio de la música de una muestra de cabeceras y búsqueda de propuestas educativas. DEDiCA. REVISTA DE EDUCAÇÃO E HUMANIDADES, 2 (2012) março, 71-86 
necesidad de utilizar escalas de medida para un nuevo paisaje sonoro (Atienza, 2007). Los cambios experimentados en las sociedades occidentales contemporáneas con la proliferación de nuevas tecnologías, hacen necesaria una nueva estrategia para proteger los derechos de los niños como ciudadanos y consumidores (Buckingham, 2002). Por ello requiere la detección de riesgos potenciales en la oferta musical televisiva y también la orientación a las audiencias, productoras, cadenas y educadores. Por último necesita de políticas para la preservación, generación y mantenimiento de contextos y micro-contextos culturales.

Este texto habla de la música en la televisión dirigida a la población infantil. Realiza una revisión del estado de la cuestión y toma como muestra las cabeceras de cuatro programas infantiles de diferentes televisiones autonómicas y nacionales en España. Finalmente muestra, como estudio de caso, el análisis de contenido de las cabeceras en comparación con un taller realizado por estudiantes de universidad. Las cabeceras de los cuatro programas han sido seleccionadas por ser un resumen de los programas que presentan, porque nos permiten identificar mediante ellas los rasgos comunes televisivos y efectos de la globalización, por ser las melodías más repetidas, utilizar varias versiones -vocales, instrumentales, completas, fraccionadas- $y$, finalmente, por indicar tendencia comunicativa en la música cotidiana dirigida al sector infantil.

\section{Fundamentación}

Una revisión de las grandes teorías en torno a la construcción del imaginario y la importancia social de la banda sonora de la televisión infantil sitúa las corrientes psicológicas, especialmente la psicología cognitiva y las aportaciones pedagógicas de la pedagogía crítica, en el eje de la discusión actual sobre el tema.

En las corrientes psicológicas destacamos las aportaciones de la Psicología Cognitiva sobre el procesamiento de la información y las teorías de la reestructuración, ambas con una clara concepción «antiasociacionista», con autores como Piaget (1969), Vygotsky (1988) o la Escuela de la Gestalt. La diferencia clave entre ellas radica en la unidad de análisis que utilizan, mientras la primera es elementarista, el enfoque cognitivo parte de unidades molares (Pozo, 1989:166-167). De ellas destacamos la teoría socio-histórica de Vygotsky, sometida a un proceso de reinterpretación y posible aplicación a la comprensión audiovisual (Korac, 1988). Vygostky 
muestra cómo los procesos mentales se explican por los instrumentos y signos que actúan de mediadores, poseedores de significado. Para Wertsch, la noción de mediación (oposredovanie) es "la contribución más original e importante de Vygotsky" (1988: 33). La argumentación principal sobre los elementos mediacionales se apoya en el estudio sobre la naturaleza comunicativa de los signos. El término signo es un concepto utilizado por Vygotsky y que identifica con ser "poseedor de significado". Según sus presupuestos es necesario que todo aquello que es interno en las formas superiores haya sido externo, es decir, que fuera para otros lo que ahora es para uno mismo. Por ello dice que toda función psicológica superior atraviesa necesariamente una etapa externa en su desarrollo, ya que inicialmente es una función social (Vygotsky, 1981: 162). De igual modo desde la Pedagogía Crítica Giroux dice que el lenguaje es el lugar de las formas reales y posibles de organización social, así como sus posibles consecuencias sociales y políticas, y todo ello se encuentra en una situación de controversia. Sin embargo, es también el lugar donde se construye nuestro sentido de nosotros mismos, nuestra subjetividad (Giroux, 1991: 76). Por ello este autor propone finalmente una recomendación educativa, y dice que ser culturalmente alfabetizado es tener la información básica necesaria para prosperar en el mundo moderno (Giroux, 1991: 43). De igual modo Buckingham (2002) habla de los cambios experimentados durante la infancia de los pobladores de las sociedades occidentales contemporáneas y señala los retos que plantea la proliferación de nuevas tecnologías, la privatización de los medios y el espacio público, así como la polarización entre los ricos y los pobres cibernéticos; argumenta que es necesaria una nueva estrategia para proteger los derechos de los niños como ciudadanos y consumidores. Desde una perspectiva pedagógica multiculturalista, los aspectos sociales que definen al "otro" , buscan entender los mecanismos de opresión y discriminación, o de libertad y reconocimiento de las minorías raciales, de clase social y de género que se reproducen a través de la construcción y transmisión de conocimientos, valores e identidades en ámbitos sociales distintos (Kincheloe, 2000). La pedagogía crítica, dice el autor, debería responder a un sistema educativo en permanente estado de confusión, donde las políticas sociales y educativas sólo interesan en términos de cómo adaptarlas a las necesidades del mercado. (Kincheloe, 2000). 
¿Qué funciones psicológicas tiene la música en la vida cotidiana? Según Hargreaves (1999), la respuesta a esta pregunta está cambiando como resultado de los actuales cambios sociales y tecnológicos en la música misma que nos obligan a re-evaluar el papel del contexto social en psicología de la música, en términos de los dominios cognitivos, emocionales y sociales. El autor concluye en que la función social de la música se manifiesta en tres formas principales para el individuo, es decir, en la gestión de la propia identidad, las relaciones interpersonales y el estado de ánimo. Esto le lleva a proponer una nueva agenda para la psicología de la música que pone la dimensión social en su núcleo, y que considera el contexto interdisciplinario, los efectos de la "democratización" de la música, el papel de la teoría, la relación entre teoría y práctica, y las implicaciones para la metodología de la investigación.

\section{Las cabeceras de los programas infantiles ${ }^{3}$}

Hemos considerado las cuatro cabeceras de los programas Babalá, emitido por Canal.9 televisión valenciana, La banda producido y emitido por Canal Sur, televisión de Andalucía, Super 3 producido y emitido por la TV3, televisión de Catalunya y Los Lunnis producido y emitido por televisión española y otros países. Todos ellos fueron emitidos en 2008 en abierto, son la cabecera de la programación infantil de tres cadenas autonómicas y una nacional. Sus características son las siguientes:

\section{La Banda. Canal Sur}

Duración 13 segundos. La música que aparece en la cabecera es la misma que se escucha de fondo durante la presentación y cierre del programa. Se trata de una melodía en ritmo binario con comienzo tético. La tonalidad es do mayor y la cadencia final de la frase es conclusiva. Con textura homofónica y sonido electrónico original, instrumental, tiene un estilo que se aproxima al tecno. La agógica depende del momento en el que se utiliza y de la función que tiene. Cuando aparece como melodía principal (cabecera) la intensidad es fuerte mientras que cuando se usa como colcha sonora (presentación y cierre) la intensidad es piano.

\section{Los Lunnis. TVE}

Se trata de una pequeña melodía con comienzo anacrúsico, tonalidad de sol Mayor a 144 negras por minuto, cantada por voces adultas y grupos vocales con acompañamiento instrumental que tiene una duración de 34". La cabecera del programa es, sin duda, la melodía que más se escucha durante las dos horas que dura el 
programa cada día de la semana, y lo hace utilizando distintas versiones: instrumental, vocal, semi hablada, completa, fraccionada, como bucle de repeticiones, etc. La composición completa, que es la que aparece al inicio del programa, tiene estructura formal que consta de una introducción, una semifrase suspensiva que se repite, una semifrase conclusiva donde queda situado el slogan del programa, y una repetición de la parte inicial con una pequeña variación en la semifrase suspensiva. La cabecera del programa termina con un cierre altamente conclusivo en el que todos los personajes gritan el nombre del programa: los Lunnis.

\section{Babalá. Canal 9. Televisión valenciana}

La programación de Babalà cuenta con un personaje que imita un gran perro que se encarga de presentar cada programa y que a su vez participa de manera vocal en la presentación o narración de muchos de los microespacios divulgativos.

\section{Club Super3. TV3}

La cabecera se utiliza en diversos momentos a lo largo de la emisión en su versión completa o fragmentada. Su estilo pertenece a pop-rock electrónico instrumental con calderón final y diminuendo que permite los enlaces entre escenas. Además se escuchan muchos ruidos sintetizados. Durante las dramatizaciones el protagonismo lo toman las voces reales de los personajes, masculinos y femeninos, con risas del público en off y efectos especiales producidos con sonidos electrónicos (glissandos, roturas...). En la autopublicidad se utilizan fragmentos de la sintonía $y$ voces en off masculinas, femeninas e infantiles.

\section{Rasgos comunes}

1. Adaptado a las secciones.

2. Ofrecen varias versiones.

3. Características musicales: Binario, tético, Mayor, electrónico.

4. Tempo entre 130 y 160 negras por minuto.

5. Duración: Entre 13 y 35 segundos.

6. Situación en el programa: Es utilizado en la mayoría de los casos como introducción y cierre.

7. Tres de los cuatro programas utilizan diferentes versiones para los apartados internos de los programas en: autopublicidad, cortinillas, presentación de contenidos, ajustes de continuidad. 


\section{Una mirada educativa sobre la banda sonora}

Música, televisión y educación tienen como elemento en común la banda sonora. Este tema en educación está contemplado en la normativa que nos proporciona la ley de educación, a través de sus leyes, decretos y normativas autonómicas. Sus referencias son el marco legal en el que la actividad didáctica tiene lugar, por lo tanto sus documentos establecen los límites posibles y también los techos, es decir los espacios imposibles, por no contemplados, en la educación musical de la educación obligatoria en España. Las referencias en materia de educación musical relacionadas con la banda sonora de la televisión aparecen en diferentes textos legales (BOE, 2003, 2006a, 2006b). Este texto se centra en las cabeceras de cuatro programas infantiles que tienen una difusión relativamente amplia por edades. Existen diferentes aportaciones en el campo de la aplicación didáctica, como los de Giráldez y en algunos monográficos de revistas como los números 23 de Comunicar y 12 de Eufonía.

Finalmente en el campo de la investigación musical los trabajos son más escasos, destacamos los estudios realizados por L. Herrera, R. Cremades y O Lorenzo (2010). Estos autores han estudiado los gustos y preferencias musicales de los niños españoles; o los de nuestro propio grupo investigador sobre el estudio de la banda sonora de la televisión infantil en una muestra latinoamericana (Porta, 2010; 2011).

Desde el punto de vista comunicativo e intercultural la música habla de uno mismo y del otro. Este aspecto desborda este pequeño texto, pero debe ser tenido en cuenta porque son necesarias aportaciones inclusivas en educación musical, siendo la cultura contemporánea y sus prácticas los principales referentes de la educación obligatoria.

\section{Aportación metodológica}

\section{¿Por qué aprender a leer la banda sonora de la televisión?}

Desde el punto de vista didáctico, todo el proceso metodológico está precedido por una pregunta: ¿Por qué aprender a leer la banda sonora de la televisión? Su respuesta tiene un planteamiento teórico que podemos resumir utilizando las palabras de Small (1989) cuando dice que "La Música es demasiado importante para dejarla en manos de los músicos". Esta afirmación tiene hoy en día varios frentes abiertos, y uno de ellos, sin duda, señala a los medios de comunicación. Y, así el autor continúa 
diciendo: "si reconocemos este hecho asestaremos un golpe al dominio que ejercen los expertos, no sólo sobre nuestra música, sino también sobre nuestra vida. Si es posible controlar nuestro propio destino musical, hacernos nuestra propia música en vez de dejar que nos la hagan otros, entonces tal vez podamos llegar igualmente a controlar a algún otro de los expertos que nos controlan la vida desde fuera".

De nuevo, observamos los extremos de una gran distancia, la que media entre la posición social, estratégica, discursiva y comunicativa de quien habla en la Música en oposición a quien escucha (Porta, 2007), porque para nosotros, siendo importante la Música, como educadores lo es más la experiencia musical. El receptor de la obra de arte, dice Small, al carecer de experiencia creativa, carece también de la necesaria confianza en su capacidad para distinguir lo que merece ser contemplado de lo que no lo merece. Al entregar a los profesionales la función creativa del arte reforzamos la tendencia cismática de nuestra sociedad, la división entre los que producen y los que consumen

\section{El proceso educativo}

Las aproximaciones al proceso educativo desde los grandes acontecimientos, soportes y contenidos a lo largo de la Historia general y también de las historias de vida, siempre han sido adaptaciones a variables en circunstancias concretas que aparecen como resolución a un problema.

Desde la Educación Musical queremos hacer un pequeño apunte, tan breve como provisional, que se centra en un recorrido educativo basado en una secuencia. Nosotros proponemos ésta (Porta, 2007b):

Expresión - Producción e Interpretación - Análisis Crítico.

Esta secuencia afectivo-cognitiva basa su consistencia en ser construida desde dentro, poco a poco, mediante la experiencia y el aprendizaje. Proponemos por ello un proceso de construcción personal que comienza con la Expresión Musical vivida en la escuela desde la inmersión cultural y el afecto, por lo tanto próxima, cotidiana y con continuidad. La segunda fase de nuestra secuencia es una consecuencia natural de la anterior, la necesidad de comunicación manifestada de forma privilegiada en la Producción e Interpretación Musical (Hacer música). Finalmente, la tercera parte de nuestra secuencia implica la reconstrucción del conocimiento mediante el Análisis Crítico cuyos resultados puedan ser, transferidos y generalizados a otros lugares y situaciones para 
poder valorar su aportación o lastre (Porta, 2007b). De esta forma, si el proceso se desarrolla en su extensión y no sólo una parte, la Música de los Mass Media en general, y la de la televisión en particular, cobrará su valor real de ser una experiencia auditiva, que podrá ser elegida o rechazada por criterios de elección en lugar de los de fascinación y seducción proporcionados por las estrategias comunicativas publicitarias y espectaculares de los grandes Medios.

\section{La construcción de significados. Las formas de lectura y el análisis de contenido}

Las tareas a realizar en la escuela relacionadas con los medios audiovisuales apuntan hacia la reconstrucción de significados a partir de los contenidos que el medio transmite (Corominas, 1999). En nuestro caso hablamos de un medio audiovisual como es la televisión que dispone de una estructura narrativa, imagen en movimiento, y también música. Gimeno Sacristán (1983) dice que el objetivo didáctico es que los alumnos adquieran un bagaje de conocimientos y estrategias cognitivas que les permitan transformar la información de que disponen en conocimientos significativos. Pérez González (1995) muestra cómo a través de los Medios de Comunicación, es posible lograr alumnos más críticos y garantizar una lectura comprensiva del lenguaje audiovisual. Sin embargo, este planteamiento centrado en el carácter mediador y la transversalidad en el currículo, tiene limitaciones cuando hablamos de la música de la televisión.

La banda sonora es uno de los componentes audiovisuales incuestionables. A través de sus contenidos y estrategias comunicativas y publicitarias construye parte del imaginario, crea opinión e invita a la acción (Porta, 2007). Por lo tanto tiene influencia y es constructor del gusto y los valores. Por ello, desde la Educación Musical las tareas centrales irán encaminadas a ofrecer metodologías de aprendizaje de lectura musical pensadas para el medio televisión y también realizar estudios de análisis de contenido sobre la misma y sus contextos culturales, narrativos y visuales que permitan crear distancia y así favorecer la postura crítica de sus contenidos, soportes, formatos y estrategias comunicativas. De esta forma será posible conocer su grado de presencia e influencia en la sociedad e intervenir desde la participación ciudadana y la educación de forma activa.

La adquisición de formas de lectura supone reforzar el área de la expresión, la escucha y el análisis. Y en ellas el papel del profesor consistirá en proporcionar los elementos necesarios para 
ayudar a reconstrucción de significados. El segundo elemento educativo musical es el Análisis de Contenido. Ferres (1996), sobre la televisión, y con anterioridad Alcocer y Úrbez (1976), sobre la lectura crítica del film, distinguen una serie de apartados. La banda sonora de la televisión requiere, igualmente, de un tratamiento metodológico adaptado al medio (Porta, 2010). Todo ello comienza por establecer escalas de medida para un nuevo contexto sonoro (Atienza, 2008). La pregunta "Qué escuchan los niños en la televisión" encierra en su respuesta una gran complejidad que gira en torno a tres sectores para su estudio (Porta, 2010):

DESCRIBIR Qué se escucha

EL RECORRIDO En qué momento se escucha

EXPERIENCIA Desde dónde se escucha.

Para realizar nuestra aportación didáctica partimos de los presupuestos expuestos, a partir de los cuales surge la necesidad de una relectura didáctica que permita dar respuesta a los nuevos problemas que plantea la lectura de la banda sonora de la televisión:

\begin{tabular}{|l|l|l|}
\hline & Desde la televisión & Desde la educación \\
\hline El contenido & Qué se escucha & Lectura musical \\
\hline El recorrido & En qué momento se escucha & $\begin{array}{l}\text { Lectura situacional y de } \\
\text { contexto }\end{array}$ \\
\hline La experiencia & Desde dónde se escucha & Lectura valorativa y crítica \\
\hline
\end{tabular}

\begin{tabular}{|c|c|}
\hline \multicolumn{2}{|r|}{ Guía de análisis } \\
\hline $\begin{array}{l}\text { Método de } \\
\text { lectura }\end{array}$ & Crear estrategias para la comprensión de la realidad \\
\hline $\begin{array}{l}\text { Lecturas } \\
\text { de la } \\
\text { diversidad }\end{array}$ & $\begin{array}{l}\text { - Procedimiento etnomusicológico, semiótico musical, estudio de } \\
\text { contextos socioculturales, la expresión y formas de } \\
\text { representación } \\
\text { - Metodología del caso único } \\
\text { - Creación de patrones: diferencias y categorías }\end{array}$ \\
\hline $\begin{array}{l}\text { Tipos de } \\
\text { textos y } \\
\text { lecturas }\end{array}$ & $\begin{array}{ll}- & \text { Lectura situacional y del contexto } \\
\text { - } & \text { Lectura musical } \\
\text { - } & \text { Lectura valorativa y crítica. }\end{array}$ \\
\hline \multicolumn{2}{|r|}{ Estrategias didácticas } \\
\hline \multicolumn{2}{|c|}{$\begin{array}{l}\text { Todas las expresivas y críticas que proporciona la Música desde la vertiente más } \\
\text { comprensiva y basada en la secuencia mencionada en el apartado anterior: } \\
\text { Expresión, Producción, Interpretación y Análisis crítico (Porta, 2007). }\end{array}$} \\
\hline
\end{tabular}

Para la Expresión disponemos de todo el bagaje adquirido a través de la obra de los grandes pedagogos de la educación musical (Willems, Dalcroze, Orff, Kodaly, Martenot, Word,...), puestas en práctica especialmente en la primera mitad del $\mathrm{S}$. XX, para la 
Producción disponemos de todos los anteriores y, además, los relacionados con la simulación de casos, los juegos de roles,...y otras que participan de la Expresión y la Producción como interpretación de obras vocales e instrumentales -aquello que llamamos "hacer música" como experiencia y, también, como producto acabado para la escucha propia y de otros: fiestas musicales, sonorizaciones, creación de programas de radio, parodias y puestas en escena de concursos... - . Se trataría de dar forma -también sonora- al principio del juego simbólico del "como si..." desarrollado por Piaget en La Psicología del Niño (Piaget, 1969) como un comienzo de comprensión y posterior análisis del fenómeno. Para el tercero de ellos, La Interpretación y el Análisis Crítico disponemos de las estrategias lingüístico-comunicativas, y discursivas, tales como: lluvia de ideas, cuestionarios semiestructurados de lápiz y papel, encuestas, sondeos de opinión, estudio y análisis de casos, análisis de contenido (Porta, 2007b).

\begin{tabular}{|c|c|c|}
\hline Método de lectura & & Análisis de contenido \\
\hline Expresión & Producción e interpretación & Análisis crítico \\
\hline $\begin{array}{l}\text { Willems, } \\
\text { Dalcroze, } \\
\text { Orff, } \\
\text { Kodaly, } \\
\text { Martenot, } \\
\text { Word, ... }\end{array}$ & $\begin{array}{c}\text { Simulación de casos, } \\
\text { Juegos de roles, ... } \\
\text { Interpretación de obras } \\
\text { vocales e instrumentales - } \\
\text { "hacer música" como } \\
\text { experiencia y como producto } \\
\text { acabado } \\
\text { Fiestas musicales, } \\
\text { Sonorizaciones, } \\
\text { Creación de programas de } \\
\text { radio, } \\
\text { Parodias y puestas en escena } \\
\text { de concursos... }\end{array}$ & $\begin{array}{c}\text { Lluvia de ideas, } \\
\text { Cuestionarios } \\
\text { semiestructurados de } \\
\text { lápiz y papel, } \\
\text { Encuestas, sondeos de } \\
\text { opinión, } \\
\text { Estudio y análisis de } \\
\text { casos, análisis de } \\
\text { contenido }\end{array}$ \\
\hline
\end{tabular}

Figura 1.- El método de lectura y el análisis de contenido

De los tres grandes apartados, el último de ellos, el Análisis Crítico es el que menos tratamiento metodológico ha recibido por parte de la educación musical. Por ello nos detendremos brevemente en él. Proponemos la lectura musical como parte central del análisis. Consta, a su vez, de tres momentos: 
1.- Lectura narrativo-musical supone considerar la música del programa de televisión en su totalidad, los personajes, el contexto de la acción y su narrativa.

2.- Lectura de la programación, programas y secciones

- Estudiar las intenciones de los productores y guionistas, bien sean explícitas o implícitas.

- Conocer los efectos del relato en el espectador individualmente o por sectores de audiencia.

- Estudiar de la banda sonora en el género audiovisual y sus peculiaridades formales, tímbricas y estilísticas

3.- Lectura crítica de la oferta televisiva, sus contenidos y valores. Requiere la implicación del profesor como mediador entre los valores transmitidos y los reconstruidos significativamente por el alumnado y responder a cuestiones tales como:

- Estudio de audiencias, contenidos y estrategias musicales. ¿A qué sectores de la población se dirige el programa y su banda sonora? ¿Qué muestra la música? ¿Qué calla?

- Estudios comparados, entre programas y entre secciones. De la misma forma proponemos estudios comparados de las tímbricas utilizadas, resoluciones y cadencias en publicidad, la elección de las tonalidades y sus cambios en las diferentes escenas de dibujos animados.

- Estudio de opciones estilísticas y sus audiencias y secciones. Géneros y estilos más/menos utilizados por las diferentes productoras, lenguas y países de procedencia, presencia de la música clásica y popular en las diferentes programaciones, proporción entre sonido sintético y acústico, efectos emocionales (por secuencias) de las bandas sonoras de películas y programas, ...

\section{Como síntesis}

Para terminar este apartado sobre la aportación metodológica al estudio de la música en la televisión infantil, recordamos las palabras de Jean Duvignaud (1972: 144) en The Sociology of Art: "La imaginación es mucho más que lo imaginario".

Por ello, para dar respuesta a las demandas de los grandes medios y soportes del S. XXI en educación musical, se hace necesario el estudio de las bandas sonoras de la televisión. Desde el punto de vista educativo quedaría inscrito en los grandes ejes de actuación de la educación contemporánea: 
1) Situar como objetivo educativo de la educación musical y su finalidad social el educar en la pluralidad de los modos de escucha.

2) Aprender a aprender:

- Problematizar el aprendizaje.

- Utilizar el aprendizaje cooperativo.

- Trabajar por proyectos y talleres.

- Creación de laboratorios sonoros.

3) La educación musical como parte del proyecto del centro.

\section{Un estudio de caso}

Presentamos una pequeña aportación, como estudio de caso, en el que hemos rastreado sobre algunos problemas planteados y su búsqueda de alternativas educativas. De forma especial queremos abordar la comparación entre la experiencia televisiva y la experiencia educativa. Presentamos a continuación la actividad titulada, "Taller de Investigación Creativa" fue realizado en diferentes sesiones entre los meses de diciembre 2010 y enero de 2011.

Creación de una ciudad sonora y/u otros

La actividad resultante del proyecto de innovación educativa realizado en la Universitat Jaume I ha sido un taller denominado Taller de Investigación Creativa, y su producto final la Creación de una ciudad sonora, realizado en diferentes sesiones entre los meses de diciembre 2010 y enero de $2011^{4}$.

Dicho taller ha tenido un carácter multidisciplinar dirigido a los siguientes profesores y estudiantes:

- Máster de Profesorado en Educación Secundaria y Bachillerato, Formación Profesional y Enseñanza de Idiomas.

- Estudiantes y profesores de la titulación de maestro Especialidad de Educación Musical.

- Estudiantes y profesores de la titulación de maestro. Especialidad Educación Infantil.

- Estudiantes y profesores de la titulación de Educación Primaria.

- Duración del taller 12 horas: Creación, preparación y realización.

Ha tenido carácter interdisciplinar en el que han participado tres áreas del Departamento de Educación, y alumnos y profesores de tres titulaciones de magisterio y del máster de secundaria. 
Ha tenido, igualmente, un carácter de innovación educativa puesto que los participantes han formado parte del diseño de la propia actividad, por medio de debates y actividades preparatorias, cuyo resultado final ha sido la construcción de un producto didáctico: "La creación de una ciudad sonora".

Finalmente podemos decir que ha sido posible sumando los esfuerzos tanto humanos como económicos de todas las partes implicadas.

Número de sesiones: El taller ha sido realizado en dos fases y cinco sesiones

\section{Creación del taller}

Sesión 1.- Diseño del taller.

Asistieron 5 profesores y 1 estudiante de la especialidad de música del master de secundaria.

Sesiones 2 y 3.- Sesión preparatoria de la plenaria del taller.

Asisten 7 estudiantes del master de Secundaria, especialidades de Plástica y Música y 2 profesores.

Fase 2

\section{Desarrollo del taller. Creación de una ciudad sonora}

Sesiones 4 y 5 .-

Duración. 2 sesiones de 3 horas.

Asistieron 12 estudiantes y profesores del Máster de Secundaria, 5 estudiantes de Magisterio Educación Infantil y 1 estudiante de Educación Musical

\section{Realización del taller. La ciudad sonora}

Soporte. Visionado de la grabación.

Clip de 10'

Inicio: Presentación de la clase.

Aparecen fragmentos del taller con sonorización de colchas. Todo a cámara rápida.

Aparecen, también a cámara rápida escenas de ciudad.

\section{Propuesta Opus $1 \quad$ 2:00 8:32}

Aparecen los tres estudiantes de secundaria de música con xilófonos.

Tocan obstinatos con los xilófonos, con efectos de agógica y dinámica. Paran y comienzan con otro motivo, mucho más lento. Alterna con imágenes a cera de vías y calles con líneas discontinuas de la calzada, ventanas, etc.

Cambian de nuevo de motivo y utilizan el efecto de golpe sobre la madera de los instrumentos. Uno de ellos añade el ruido de los zapatos al caminar. También añaden el frotado con el mango de la baqueta.

Suena después la secuencia clásica de la hora en un reloj antiguo de pared en un carillón al que se unen todos con un efecto de contraste muy desordenado y simultáneo utilizando tres instrumentos.

Comienzan con un latido que después va acelerando, se suman otros, utilizan diferentes sonoridades de los xilófonos y añaden obstinatos muy repetitivos con instrumentos de percusión como muelles, tambores, batidores hasta quedar sólo el bombo. 
Después surge el piano e interpreta una melodía de corte jazzístico bastante melódico, de aire ambiental. Cambia a un diseño melódico un poco más atrevido y menos atado a la tonalidad con algunas disonancias.

\section{Propuesta Opus 2 9:00}

Grupo de xilófonos. Participan estudiantes de maestro Educación Infantil y del máster de Secundaria de Plástica

Hablan y tocan los instrumentos simultáneamente, creando con ellos secuencias y motivos no melódicos.

Usan el piano, crean diseños no melódicos, glissandos y efectos secos de piano. El conductor de la sesión añade sonidos como de agua, pájaros.

Del conjunto surge un obstinato, con el que se identifican como eje formal, melódico y rítmico todo el conjunto de participantes.

Sobreimpresionado aparecen y desaparecen dibujos infantiles de la ciudad con técnica de ceras. Mucho colorido: vías con líneas discontinuas del centro de la calzada, señales

Paran todos, comienza uno de ellos a frotar con la mano abierta un pandero.

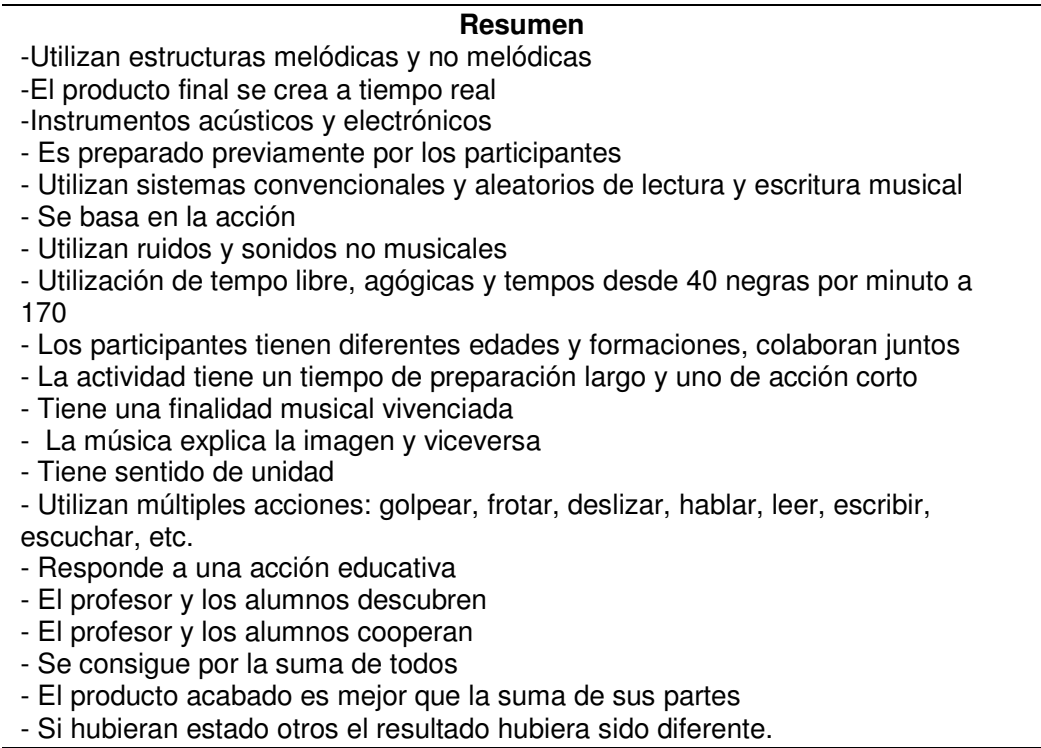

\section{Referencias}

Aronowitz, S.; Giroux, H. A. (1991). Postmodern education. Politics, culture and social criticism. Minneapolis: University of Minnesota Press.

Atienza, R. (2008). L'identité sonore: une variable essentielle dans la configuration urbaine. Cresson: Grenoble.

Boletín Oficial del Estado (2003). RD 943/2003, BOE 31/07/2003.

Boletín Oficial del Estado (2006a). LO2/2006), II, 1, 71,76, 77.

Boletín Oficial del Estado (2006b). RD1513/2006, de 7/12/2006. 
Buckingham, D. (2002). Crecer en la era de los medios electrónicos. Madrid: Morata.

Collins, W. A.; Korac. N (1982). Recent progressism in the study of the effects of television viewing on social development. International Journal of Behavioural Development, 5 (1982) 171-193.

Duvignaud, J. (1972). The Sociology of Art. London: Paladin.

Ferrés, J. (1996). Televisión subliminal. Socialización mediante comunicaciones inadvertidas. Barcelona: Paidós.

Gimeno Sacristán, J.; Pérez Gómez, A. (1983). La enseñanza: su teoría y su práctica. Madrid: Akal.

Giráldez, A. (2005). Internet y educación musical. Barcelona: Graó.

Hargreaves, D. J.; North, A. C. (1999). The functions of music in everyday life: Redefining the social in music psychology. Psychology of Music, 27, 1 (1999) 71-83.

Herrera, L.; Cremades, R.; and Lorenzo, O. (2010). Musical preferences in compulsory secondary education students: Influence of formal and informal education. Cultura y Educación, 22, 1 (2010) 37-51.

Kincheloe, J. L.; Steinberg, S. R.; Real, J. 2000). Repensar el multiculturalismo. Barcelona. Octaedro.

Korac, N. (1988). Los medios de comunicación visual y el desarrollo cognoscitivo. Belgrado: Universidad de Belgrado.

Piaget, J. (1969). Psicología del niño. Madrid: Morata.

Porta, A. (2007). Músicas públicas, escuchas privadas. Hacia una lectura de la música popular contemporánea. Aldea Global. Barcelona: UAB.

Porta, A.; Ferrández, R. (2009). Elaboración de un instrumento para conocer las características de la banda sonora de la programación infantil de televisión. RELIEVE, 15, 2 (2009). Disponible en: http://www.uv.es/RELIEVE/v15n2/RELIEVEv15n2_6.htm Consultado en 11/11/2010. UJI/Rivera.

Porta, A. (2010). ¿Qué escuchan los niños en la TV? Castellón: Morata.

Pozo, J. I. (1989). Teorías cognitivas del aprendizaje. Madrid:

Small, C. (1989). Música, Sociedad y Educación. Madrid: Alianza.

VVAA (2004). Comunicación, Música y tecnologías. Comunicar, 23 (2004)10-60.

VVAA (1998). № monográfico Música moderna. Eufonía, 12 (1998).

Vygotsky, L. (1981). The instrumental method in psychology. En J. Wertsch (Ed.), The concept of activity in Soviet Psychology, 134-143. New York: Sharpe.

Wertsch, J. (1988). Vigotsky y la formación social de la mente. Barcelona: Paidós.

Wolfe, D. E.; Stambaugh, S. (1993). Musical analysis of Sesame Street: Implications for music therapy practice and research. Journal of Music Therapy, 30, 4 (1993) 224-235. 
${ }^{1}$ Educational interpretation of television: A study of the music in a sample of opening themes and educational proposals

2 Doctora.

Universitat Jaume I (España).

Email: porta@edu.uji.es

${ }^{3}$ Estas descripciones fueron realizadas por los siguientes autores: Babalá. Canal 9 por Remigi Morant. Universidad de Valencia, Super 3 por Olga González y Josep Gustems. Universidad de Barcelona, La Banda. Canal Sur por Almudena Ocaña y $M^{a}$ Luisa Reyes. Los Lunnis TVE. Amparo Porta. Disponibles en Porta (Ed.) (2010) ¿Qué escuchan los niños en la televisión? UJI/Rivera.

${ }^{4}$ El taller fue llevado a cabo por el profesor Adolf Murillo. Profesor de Secundaria y director de "Com sona l'Eso". 